\title{
Effect of Time and Legume Type on Germination-Induced Proteolysis of Lentils and Faba Beans ${ }^{\dagger}$
}

\author{
Sara Bautista-Expósito ${ }^{1}$, Elena Peñas ${ }^{1}$, Albert Vanderberg ${ }^{2}$, Juana Frias ${ }^{1}$ and Cristina Martínez-Villaluenga ${ }^{1, *}$ \\ 1 Department of Food Characterization, Quality and Safety, Institute of Food Science, Technology and \\ Nutrition (ICTAN-CSIC), 28040 Madrid, Spain; sara.bautista@ictan.csic.es (S.B.-E.); \\ elenape@ictan.csic.es (E.P.); frias@ictan.csic.es (J.F.) \\ 2 Department of Plant Sciences, University of Saskatchewan, Saskatoon, SK S7N 5A8, Canada; \\ bert.vandenberg@usask.ca \\ * Correspondence: c.m.villaluenga@csic.es; Tel.: +34-912587601 \\ + Presented at the 1st International Electronic Conference on Food Science and Functional Foods, \\ 10-25 November 2020; Available online: https://foods_2020.sciforum.net/.
}

Citation: Bautista-Expósito, S.;

Peñas, E.; Vanderberg, A.; Frias, J.; Martínez-Villaluenga, C. Effect of Time and Legume Type on Germination-Induced Proteolysis of Lentils and Faba Bean. Proceedings 2021, 70, 4. https://doi.org/10.3390/ foods_2020-07823

Published: 10 November 2020

Publisher's Note: MDPI stays neutral with regard to jurisdictional claims in published maps and institutional affiliations.

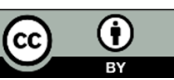

Copyright: (c) 2020 by the authors. Licensee MDPI, Basel, Switzerland. This article is an open access article distributed under the terms and conditions of the Creative Commons Attribution (CC BY) license (http://creativecommons.org/licenses/by/4.0/).

\begin{abstract}
Legumes are alternative protein sources that have been successfully used to develop diverse meatless foods. Although these plant-based products have a lower impact on the environment than equivalent animal-based products, they have lower protein digestibility. Germination could be a useful bioprocess to enhance protein digestibility in legumes, although its effect at different times of seedling development has been little studied in lentils and faba beans. This work investigated the effect of germination time ( 4 and 6 days after full seed imbibition) on the proteins of three types of Canadian lentils ("gray zero tannin", G; "caviar black", B; and "red dehulled", D) and faba beans ("zero vicin/convicin", F). Germination increased total nitrogen (4-14\% increase) and total levels of some amino acids: Asp in all the sprouts studied; Ser, Pro, Ala, Cys, His and Lys in G; and Met and Tyr in B. A concurrent degradation of the 7S and 11S globulin subunits, the accumulation of peptides below $20 \mathrm{kDa}$ and free essential and non-essential amino acids (4- to 6-fold increase) were observed after germination in all the legumes studied. These effects were attributable to the increased protease activity observed after sprouting. Trypsin inhibitory activity was lower in legume sprouts, except for D, where a small increase was detected. Time, legume type and their interaction showed significant effects on the parameters studied. Germination effects were generally more remarkable at longer stages of seedling development. Among the legumes studied, D showed a differential behavior characterized by a faster protein degradation and release of small peptides, probably due to its higher protease activity as indicated by principal component analysis. These results evidence the positive effects of germination on the protein digestibility of different lentil types and faba beans. The protein quality of plant-based foods could be improved through the selection of legume species with higher germination-induced proteolytic rates and optimized germination times.
\end{abstract}

Keywords: Lentil, faba bean, germination, protein, hydrolysis

Supplementary Materials: The following are available online at https://www.mdpi.com/25043900/70/1/4/s1.

Funding: This research was funded by the University of Saskatchewan, grant "Lentils for Sustain

Institutional Review Board Statement: In this section, you should add the Institutional Review Board Statement and approval number, if relevant to your study. You might choose to exclude this statement if the study did not require ethical approval. Please note that the Editorial Office might ask you for further information. Please add "The study was conducted according to the guidelines of the Declaration of Helsinki, and approved by the Institutional Review Board (or Ethics Committee) of NAME OF INSTITUTE (protocol code XXX and date of approval)." OR "Ethical review and approval were waived for this study, due to REASON (please provide a detailed justification)." OR "Not applicable" for studies not involving humans or animals. 
Informed Consent Statement: Please add "Informed consent was obtained from all subjects involved in the study." OR "Patient consent was waived due to REASON (please provide a detailed justification)." OR "Not applicable" for studies not involving humans.

Data Availability Statement: Please refer to suggested Data Availability Statements in section "MDPI Research Data Policies" at https://www.mdpi.com/ethics. 